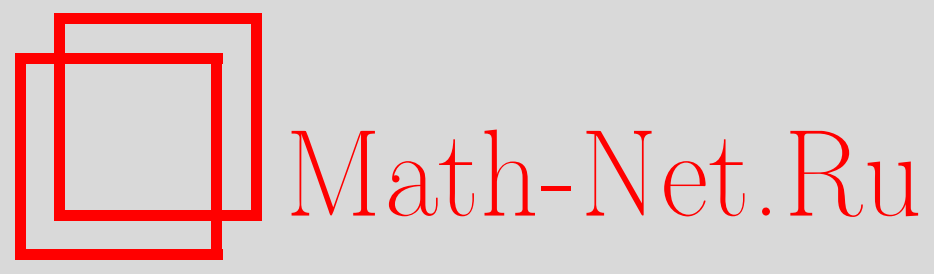

Г. А. Карпунин, И. Г. Шапошников, Скрещенные гомоморфизмы конечных многоосновных универсальных алгебр с бинарными операциями, Дискрет. матем., 2000, том 12, выпуск 2, 66-84

DOI: https://doi.org/10.4213/dm331

Использование Общероссийского математического портала Math-Net.Ru подразумевает, что вы прочитали и согласны с пользовательским соглашением http://www.mathnet.ru/rus/agreement

Параметры загрузки:

IP : 34.239 .49 .27

26 апреля 2023 г., 13:01:28

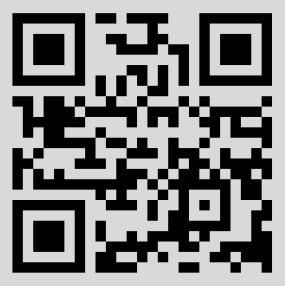




\title{
Скрещенные гомоморфизмы конечных многоосновных универсальных алгебр с бинарными операциями
}

\author{
(c) 2000 г. $\quad$ Г. А. Карпунин, И. Г. Шапошников
}

\begin{abstract}
Вводится понятие правого (левого) скрещенного гомоморфизма конечных многоосновных универсальных алгебр с бинарными операциями, обобщающее понятие правой (левой) скрещенной изотопии квазигрупп, введенных В. Д. Белоусовым. Доказывается теорема о скрещенных гомоморфизмах (аналог классической теоремы о гомоморфизмах). Описание скрещенных гомоморфизмов многоосновной универсальной алгебры сводится к описанию ее скрещенных конгруэнций. Изучаются скрещенные конгруэнции квазигрупп, изотопных группам и скрещенно изотопных группам. Показана возможность использования скрещенных конгруэнций для постороения алгоритмов решения уравнений над алгебрами.
\end{abstract}

\section{1. Введение}

В работе В. Д. Белоусова [1] было введено понятие скрещенной изотопии квазигрупп. Напомним его определение. Тройка $T=(\alpha, B, \gamma)$, где $\alpha, \gamma$ - подстановки, а $B-$ обратимая справа операция на множестве $Q$, называется правой скрещенной изотопией или $R$-изотопией, а квазигруппа $Q(*)$ называется $R$-изотопной квазигруппе $Q(\cdot)$, если

$$
x * y=\gamma^{-1}(\alpha x \cdot B(x, y))
$$

для всех $x, y \in Q$.

Аналогично определяется левая скрещенная изотопия или $L$-изотопия $S=$ $(A, \beta, \gamma)$, где $\beta, \gamma$ - подстановки, а $A$ - обратимая слева операция на множестве $Q$. Квазигруппа $Q(*)$ называется $L$-изотопной квазигруппе $Q(\cdot)$, если существует такая $L$-изотопия $S=(A, \beta, \gamma)$, что

$$
x * y=\gamma^{-1}(A(x, y) \cdot \beta y)
$$

для всех $x, y \in Q$.

Скрещенные изотопы появляются в ряде практических приложений, например, в криптографии. Известно, что, как правило, алгоритмы блочного шифрования строятся по так называемому принципу Feistel networks [2], по которому шифруемый 
блок длины $2 n$ двоичных знаков разбивается на равные части $L_{0}$ и $R_{0}$ и преобразуется в течении ряда однотипных циклов по следующему закону в $i$-м цикле:

$$
\begin{aligned}
& L_{i}=R_{i-1}, \\
& R_{i}=L_{i-1} \oplus f\left(R_{i-1}, K_{i}\right),
\end{aligned}
$$

где $K_{i}$ - элемент ключа, $f$ - некоторая функция, $\oplus$ - покоординатное сложение двоичных векторов по модулю 2 . Если $L_{i}, R_{i}, K_{i}$ - элементы одного множества $V_{n}$, а $f$ является обратимой справа операцией на $V_{n}$, то нетрудно показать, что операция

$$
F: V_{2 n} \times V_{2 n} \rightarrow V_{2 n}
$$

преобразования блока за два цикла:

$$
F\left(\left(L_{i-1}, R_{i-1}\right),\left(K_{i}, K_{i+1}\right)\right)=\left(L_{i+1}, R_{i+1}\right),
$$

задает структуру квазигруппы $V_{2 n}(F), R$-изотопной группе $V_{2 n}(\oplus)$ при $R$-изотопии $(e, B, e)$, где $e$ - тождественное отображение, а $B$ имеет вид

$$
B\left(\left(L_{i-1}, R_{i-1}\right),\left(K_{i}, K_{i+1}\right)\right)=\left(f\left(R_{i-1}, K_{i}\right), f\left(L_{i-1} \oplus f\left(R_{i-1}, K_{i}\right), K_{i+1}\right)\right) .
$$

Изотопии квазигрупп, в том числе и скрещенные изотопии, могут быть рассмотрены как частный случай гомотопии (соответственно скрещенной гомотопии) квазигрупп. Скрещенную гомотопию мы определим следующим образом.

Определение 1. Рассмотрим квазигруппы $Q(*)$ и $P(\cdot)$. Тройку $T=(\alpha, B, \gamma)$, где $\alpha$, $\gamma$ - отображения $Q$ в $P$, а $B$ - отображение $P \times Q$ в $P$, назовем правой скрещенной гомотопией или $R$-гомотопией, если

$$
\gamma(x * y)=\alpha x \cdot B(\alpha x, y)
$$

для всех $x, y \in Q$. При этом квазигруппа $Q(*)$ называется $R$-гомотопной квазигруппе $P(\cdot)$.

Аналогично определяется левая скрещенная гомотопия или $L$-гомотопия $S=$ $(A, \beta, \gamma)$, где $\beta, \gamma$ - отображения $Q$ в $P$, а $A$ - отображение $Q \times P$ в $P$. Квазигруппа $Q(*)$ называется $L$-гомотопной квазигруппе $P(\cdot)$, если существует такая $L$-гомотопия $S=(A, \beta, \gamma)$, что

$$
\gamma(x * y)=A(x, \beta y) \cdot \beta y
$$

для всех $x, y \in Q$.

В представленной статье развиваются идеи В. Д. Белоусова по построению и исследованию скрещенных изоморфизмов и гомоморфизмов конечных многоосновных универсальных алгебр с бинарными операциями, то есть пар $(\mathfrak{M} ; \Sigma)$, где $\mathfrak{M}$ - семейство осно́вных конечных множеств, а $\Sigma$ - семейство бинарных операций, определенных на элементах $\mathfrak{M}$. Скрещенные изоморфизмы и гомоморфизмы многоосновных универсальных алгебр с бинарными операциями являются обобщениями скрещенных изотопий и гомотопий квазигрупп, определенных выше, а также обобщениями изоморфизмов и гомоморфизмов многоосновных универсальных алгебр, рассмотренных в классической работе Хиггинса [3]. Такое направление исследований представляет не только теоретический, но и практическое интерес, например, для решения уравнений над алгебрами. Основным объектом исследования статьи 
является скрещенный гомоморфизм многоосновной универсальной алгебры с одной бинарной операцией. Полученные результаты естественным образом распространяются на случай произвольных многоосновных универсальных алгебр с бинарными операциями.

Многоосновную универсальную алгебру с одной бинарной операцией $F: A \times B \rightarrow$ $C$ будем обозначать через $(A, B, C ; F)$. Поскольку других алгебр кроме многоосновных универсальных у нас не появится, то два этих прилагательных мы, как правило, будем опускать. Пусть у алгебры $\mathscr{A}=(A, B, C ; F)$ операция $F$ частично определена. Через $D(F)$ будем обозначать множество упорядоченных троек элементов $(a, b, c) \in(A, B, C)$ таких, что на $(a, b)$ определена операция $F$ и $F(a, b)=c$. Множество $D(F)$ назовем оператой алгебры $\mathscr{A}$.

Рассмотрим две алгебры

$$
\mathscr{A}_{1}=\left(A_{1}, B_{1}, C_{1} ; F\right), \quad \mathscr{A}_{2}=\left(A_{2}, B_{2}, C_{2} ; H\right)
$$

с частичными бинарными операциями

$$
F: A_{1} \times B_{1} \rightarrow C_{1}, \quad H: A_{2} \times B_{2} \rightarrow C_{2}
$$

соответственно.

Определение 2. Пусть тройка $(\alpha, B, \Gamma)$, состоящая из набора отображений

$$
\alpha: A_{1} \rightarrow A_{2}, \quad B: A_{2} \times B_{1} \rightarrow B_{2}, \quad \Gamma: A_{2} \times C_{1} \rightarrow C_{2},
$$

задает отображение $T$ операты $D(F)$ алгебры $\mathscr{A}_{1}$ в операту $D(H)$ алгебры $\mathscr{A}_{2}$ следующим образом:

$$
T(a, b, c)=(\alpha(a), B(\alpha(a), b), \Gamma(\alpha(a), c))
$$

то есть

$$
H(\alpha(a), B(\alpha(a), b))=\Gamma(\alpha(a), F(a, b))
$$

Тогда будем говорить, что тройка $(\alpha, B, \Gamma)$ является правым скрещенным гомоморфизмом алгебры $\mathscr{A}_{1}$ в алгебру $\mathscr{A}_{2}$.

Определение 3. Аналогично определяется понятие левого скрещенного гомоморфизма. Пусть тройка $(A, \beta, \Gamma)$, состоящая из набора отображений

$$
\beta: B_{1} \rightarrow B_{2}, \quad A: B_{2} \times A_{1} \rightarrow A_{2}, \quad \Gamma: B_{2} \times C_{1} \rightarrow C_{2}
$$

задает отображение $T$ операты $D(F)$ алгебры $\mathscr{A}_{1}$ в операту $D(H)$ алгебры $\mathscr{A}_{2}$ следующим образом:

$$
T(a, b, c)=(A(\beta(b), a)), \beta(b), \Gamma(\beta(b), c))
$$

то есть

$$
H(A(\beta(b), a), \beta(b))=\Gamma(\beta(b), F(a, b)) .
$$

Тогда будем говорить, что тройка $(A, \beta, \Gamma)$ является левым скрещенным гомоморфизмом алгебры $\mathscr{A}_{1}$ в алгебру $\mathscr{A}_{2}$. 
Если в выше приведенных определениях скрещенных гомоморфизмов отображения $\alpha, \beta$ биективны и биективны отображения $B, A, \Gamma$ по второму аргументу при фиксированном первом, то такие скрещенные гомоморфизмы являются соответственно правым и левым скрещенным изоморфизмом алгебры $\mathscr{A}_{1}$ и алгебры $\mathscr{A}_{2}$. Если, кроме того, отображения $B, A, \Gamma$ не зависят от множеств соответственно $A_{2}$, $B_{2}$ и $A_{2}$ (или $B_{2}$ ), то мы как частный случай получаем определение изоморфизма многоосновных универсальных алгебр с частичными операциями.

В дальнейшем будем рассматривать только правые скрещенные гомоморфизмы и изоморфизмы. Поэтому мы, как правило, будем опускать прилагательное правое для связанных с ним понятий. Все полученные результаты для правых скрещенных гомоморфизмов и изоморфизмов естественным образом переносятся на левые скрещенные гомоморфизмы и изоморфизмы. Ситуация аналогична и для других далее вводимых понятий.

\section{2. Скрещенные конгруэнции}

Определение 4. Совокупность отношений эквивалентности

$$
\varepsilon=\left(\varepsilon_{A},\left\{\varepsilon_{[a]}:[a] \in A / \varepsilon_{A}\right\},\left\{\gamma_{[a]}:[a] \in A / \varepsilon_{A}\right\}\right)
$$

на основных множествах $A, B, C$ соответственно будем называть правой скрещенной конгруэнцией на алгебре $(A, B, C ; F)$, если выполняется условие: для любых классов эквивалентности $[a] \varepsilon_{A}$ и $[b] \varepsilon_{[a]}$ существует класс $[c] \gamma_{[a]}$ такой, что имеет место включение

$$
F\left([a] \varepsilon_{A},[b] \varepsilon_{[a]}\right) \subset[c] \gamma_{[a]} .
$$

Аналогично определяется левая скрещенная конгруэнция.

Иногда вместо символов, обозначающих отношения эквивалентности, мы будем указывать соответствующие классы эквивалентности, что не должно вызвать недоразумений.

Пусть

$$
T=(\alpha, B, \Gamma): \mathscr{A}=(A, B, C ; F) \rightarrow \mathscr{A}^{\prime}=\left(A^{\prime}, B^{\prime}, C^{\prime} ; F^{\prime}\right)
$$

- скрещенный гомоморфизм. Под $\operatorname{ker} T$ будем понимать набор эквивалентностей

$$
\left(\varepsilon_{A},\left\{\varepsilon_{[a]}:[a] \in A / \varepsilon_{A}\right\},\left\{\gamma_{[a]}:[a] \in A / \varepsilon_{A}\right\}\right),
$$

заданных следующим образом:

$$
\begin{aligned}
& \varepsilon_{A}: a, a^{\prime} \in A, a \sim a^{\prime} \Longleftrightarrow \quad \alpha(a)=\alpha\left(a^{\prime}\right) ; \\
& \varepsilon_{[a]}: b, b^{\prime} \in B, b \sim b^{\prime} \Longleftrightarrow B(\alpha(a), b)=B\left(\alpha(a), b^{\prime}\right) ; \\
& \gamma_{[a]}: c, c^{\prime} \in C, c \sim c^{\prime} \Longleftrightarrow \Gamma(\alpha(a), c)=\Gamma\left(\alpha(a), c^{\prime}\right) .
\end{aligned}
$$

Легко проверить, что $\operatorname{ker} T$ является скрещенной конгруэнцией на алгебре $\mathscr{A}$.

Для любой скрещенной конгруэнции $\varepsilon$ на алгебре $\mathscr{A}$ можно построить факторалгебру $\widetilde{\mathscr{A}}=\mathscr{A} / \varepsilon$. Опишем эту процедуру. В качестве основных множеств для фактор-алгебры $\widetilde{\mathscr{A}}=\mathscr{A} / \varepsilon$ возьмем множества

$$
\widetilde{A}=A / \varepsilon_{A}, \quad \widetilde{B}=\left\{\cup B / \varepsilon_{[a]}:[a] \in A / \varepsilon_{A}\right\}, \quad \widetilde{C}=\left\{\cup C / \gamma_{[a]}:[a] \in A / \varepsilon_{A}\right\} .
$$


Определим теперь на этих множествах частичную операцию $\widetilde{F}$ следующим образом. Операция $\widetilde{F}$ задается только на парах вида $\left([a] \varepsilon_{A},[b] \varepsilon_{[a]}\right)$. Поскольку $\varepsilon$ - скрещенная конгруэнция, для любой такой пары найдется единственный класс $[c] \gamma_{[a]}$ такой, что выполняется соотношение (3). Положим

$$
\widetilde{F}\left([a] \varepsilon_{A},[b] \varepsilon_{[a]}\right)=[c] \gamma_{[a]} .
$$

Алгебру $\widetilde{\mathscr{A}}=(\widetilde{A}, \widetilde{B}, \widetilde{C} ; \widetilde{F})$ назовем фактор-алгеброй алгебры $\mathscr{A}$ по скрещенной конгруэнции $\varepsilon$.

Определим естественный скрещенный гомоморфизм $\widetilde{T}=(\widetilde{\alpha}, \widetilde{B}, \widetilde{\Gamma})$ из алгебры $\mathscr{A}$ в фактор-алгебру $\widetilde{\mathscr{A}}=\mathscr{A} / \varepsilon$ следующим образом:

$$
\begin{array}{rrrr}
\widetilde{\alpha}: & A \rightarrow \widetilde{A}, & \widetilde{\alpha}(a) & =[a] ; \\
\widetilde{B}: \widetilde{A} \times B \rightarrow \widetilde{B}, & \widetilde{B}([a], b)=[b] \varepsilon_{[a]} ; \\
\widetilde{\Gamma}: \widetilde{A} \times C \rightarrow \widetilde{C}, & \widetilde{\Gamma}([a], c)=[c] \gamma_{[a]} .
\end{array}
$$

При так определенных отображениях $\widetilde{\alpha}, \widetilde{B}, \widetilde{\Gamma}$ для любых $a \in A, b \in B$ выполняется равенство

$$
\widetilde{F}(\widetilde{\alpha}(a), \widetilde{B}(\widetilde{\alpha}(a), b))=\widetilde{\Gamma}(\widetilde{\alpha}(a), F(a, b)) .
$$

Следовательно, $\widetilde{T}=(\widetilde{\alpha}, \widetilde{B}, \widetilde{\Gamma})$ на самом деле является скрещенным гомоморфизмом.

Пусть

$$
T=(\alpha, B, \Gamma): \mathscr{A}=(A, B, C ; F) \rightarrow \mathscr{A}^{\prime}=\left(A^{\prime}, B^{\prime}, C^{\prime} ; F^{\prime}\right)
$$

- скрещенный гомоморфизм. Образом $\operatorname{Im}(\alpha, B, \Gamma)$ гомоморфизма $T$ будем называть алгебру $\mathscr{A}_{0}^{\prime}=\left(A_{0}^{\prime}, B_{0}^{\prime}, C_{0}^{\prime} ; F^{\prime}\right)$, где

$$
\begin{aligned}
A_{0}^{\prime} & =\operatorname{Im} \alpha=\left\{a^{\prime} \in A^{\prime}: \exists a \in A, \alpha(a)=a^{\prime}\right\} \\
B_{0}^{\prime} & =\bigcup_{a \in A} \operatorname{Im} B(\alpha(a), \cdot)=\bigcup_{a^{\prime} \in A_{0}^{\prime}} \operatorname{Im} B\left(a^{\prime}, \cdot\right) \\
& =\bigcup_{a^{\prime} \in A_{0}^{\prime}}\left\{b^{\prime} \in B^{\prime}: \exists b \in B, B\left(a^{\prime}, b\right)=b^{\prime}\right\}, \\
C_{0}^{\prime} & =\bigcup_{a \in A} \operatorname{Im} \Gamma(\alpha(a), \cdot)=\bigcup_{a^{\prime} \in A_{0}^{\prime}} \operatorname{Im} \Gamma\left(a^{\prime}, \cdot\right) \\
& =\bigcup_{a^{\prime} \in A_{0}^{\prime}}\left\{c^{\prime} \in C^{\prime}: \exists c \in C, \Gamma\left(a^{\prime}, c\right)=c^{\prime}\right\},
\end{aligned}
$$

а операция $F^{\prime}$ на алгебре $\mathscr{A}_{0}^{\prime}$ индуцируется с алгебры $\mathscr{A}^{\prime}$.

Лемма 1. Скрещеннъй гомоморфизм алгебры $(A, B, C ; F)$ в нехоторую алгебру существует тогда и только тогда, когда на основньх множествах $A, B$ и $C$ соответственно определень отночения эквивалентности $\varepsilon_{A}$,

$$
\left\{\varepsilon_{[a]}:[a] \in A / \varepsilon_{A}\right\}, \quad\left\{\gamma_{[a]}:[a] \in A / \varepsilon_{A}\right\}
$$

образующие скрещенную конгруэнцию на алгебре $(A, B, C ; F)$. 
Дохазательство. Докажем необходимость. Если существует скрещенный гомоморфизм $T=(\alpha, B, \Gamma)$ алгебры $(A, B, C ; F)$ в некоторую алгебру, то в качестве скрещенной конгруэнции на алгебре $(A, B, C ; F)$ можно взять $\operatorname{ker} T$.

Докажем достаточность. Если существует скрещенная конгруэнция $\varepsilon$ на алгебре $\mathscr{A}=(A, B, C ; F)$, тогда построим фактор-алгебру $\widetilde{\mathscr{A}}=\mathscr{A} / \varepsilon$ и возьмем естественный скрещенный гомоморфизм $\widetilde{T}=(\widetilde{\alpha}, \widetilde{B}, \widetilde{\Gamma})$ из $\mathscr{A}$ в $\widetilde{\mathscr{A}}$.

Теорема 1. Образ скрещенного гомоморфизма $T: \mathscr{A} \rightarrow \mathscr{A}^{\prime}$ изоморфен факторалгебре $\mathscr{A} / \operatorname{ker} T$.

Доказателъство. Построим гомоморфизм $\left(\widetilde{\alpha}_{0}, \widetilde{\beta}_{0}, \widetilde{\gamma}_{0}\right)$ из фактор-алгебры $\widetilde{\mathscr{A}}=$ $\mathscr{A} / \operatorname{ker} T$ в алгебру $\operatorname{Im}(\alpha, B, \Gamma)=\left(A_{0}^{\prime}, B_{0}^{\prime}, C_{0}^{\prime} ; F^{\prime}\right)$ следующим образом:

$$
\begin{array}{rlrl}
\widetilde{\alpha}_{0}: \tilde{A} \rightarrow A_{0}^{\prime}, & \widetilde{\alpha}_{0}([a])=\alpha(a) ; \\
\widetilde{\beta}_{0}: \widetilde{B} \rightarrow B_{0}^{\prime}, & & \widetilde{\beta}_{0}\left([b] \varepsilon_{[a]}\right)=B(\alpha(a), b) ; \\
\widetilde{\gamma}_{0}: \widetilde{C} \rightarrow C_{0}^{\prime}, & & \widetilde{\gamma}_{0}\left([c] \gamma_{[a]}\right)=\Gamma(\alpha(a), c) .
\end{array}
$$

Согласно определению $\operatorname{ker} T$ и $\widetilde{\mathscr{A}}=\mathscr{A} / \operatorname{ker} T$ отображения $\widetilde{\alpha}_{0}, \widetilde{\beta}_{0}, \widetilde{\gamma}_{0}$ корректно определены. Используя равенство (2) для скрещенного гомоморфизма $(\alpha, B, \Gamma)$, непосредственно можно убедиться, что для любой пары $[a] \in \widetilde{A},[b] \varepsilon_{[a]} \in \widetilde{B}$ выполняется соотношение

$$
F^{\prime}\left(\widetilde{\alpha}_{0}([a]), \tilde{\beta}_{0}\left([b] \varepsilon_{[a]}\right)\right)=\tilde{\gamma}_{0}\left(\widetilde{F}\left([a],[b] \varepsilon_{[a]}\right)\right),
$$

то есть $\left(\widetilde{\alpha}_{0}, \widetilde{\beta}_{0}, \widetilde{\gamma}_{0}\right)$ является гомоморфизмом. Нетрудно проверить, что построенные отображения $\widetilde{\alpha}_{0}, \widetilde{\beta}_{0} ; \widetilde{\gamma}_{0}$ удовлетворяют условиям из определения изоморфизма, и

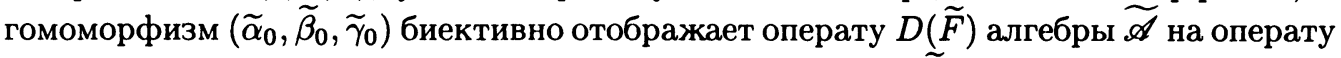
$D\left(F^{\prime}\right)$ алгебры $\mathscr{A}_{0}^{\prime}=\left(A_{0}^{\prime}, B_{0}^{\prime}, C_{0}^{\prime} ; F^{\prime}\right)$. Следовательно, $\left(\widetilde{\alpha}_{0}, \widetilde{\beta}_{0}, \widetilde{\gamma}_{0}\right)-$ изоморфизм.

Согласно лемме 1 и теореме 1 описание всех скрещенных конгруэнций на алгебре $\mathscr{A}=(A, B, C ; F)$ эквивалентно описанию с точностью до изоморфизма ее скрещенно гомоморфных образов. Поэтому далее мы будем исследовать скрещенные конгруэнции, так как в этом случае мы ограничиваемся исследованием свойств операций алгебры.

\section{3. Алгоритм решения уравнений над алгебрами}

Известны алгоритмы решения систем уравнений над алгебрами, использующие существование гомоморфизмов (см., например, $[4,5,6,7])$. Аналогичным образом можно использовать и скрещенные гомоморфизмы. Рассмотрим, например, уравнение над алгеброй $(A, B, C ; F)$ с одной бинарной операцией $F$ относительно неизвестных $a \in A$ и $b \in B$ вида

$$
F(a, b)=c, \quad c \in C
$$

Пусть на алгебре $(A, B, C ; F)$ существует некоторая скрещенная конгруэнция

$$
\varepsilon=\left(\varepsilon_{A},\left\{\varepsilon_{[a]}:[a] \in A / \varepsilon_{A}\right\},\left\{\gamma_{[a]}:[a] \in A / \varepsilon_{A}\right\}\right)
$$


Тогда, используя ее, можно свести решение уравнения (4) к решению уравнения в скрещенной фактор-алгебре вида

$$
\widetilde{F}\left([a],[b] \varepsilon_{[a]}\right)=[c] \gamma_{[a]} \cdot
$$

Уравнение (5) может решаться проще уравнения (4), хотя бы за счет меньшей мощности множества неизвестных. Например, простейшим алгоритмом решения уравнения (4) с использованием скрещенной конгруэнции $\varepsilon$ является алгоритм перебора представителей классов эквивалентности $[a],[b] \varepsilon_{[a]}$ на первом этапе и перебора элементов внутри классов эквивалентности, для которых выполняется соотношение (5), на втором этапе. Вычислительная сложность решения уравнения (4) данным алгоритмом составляет величину

$$
Q(\varepsilon)=\sum_{[a] \in A / \varepsilon_{A}}\left|B / \varepsilon_{[a]}\right|+\sum|[a]|\left|\left[b_{s}\right] \varepsilon_{[a]}\right|,
$$

где суммирование во втором слагаемом производится по представителям классов $a$, $b_{s}$ таким, что

$$
F\left([a],\left[b_{s}\right] \varepsilon_{[a]}\right) \subset[c] \gamma_{[a]} .
$$

Таким образом, принцип использования скрещенных гомоморфизмов и скрещенных конгруэнций для решения систем уравнений над алгебрами, аналогичен принципу использования традиционных гомоморфизмов и конгруэнций $[4,8]$. Вместе с тем, за счет большей общности понятия скрещенной конгруэнции, алгоритм решения уравнений с их использованием может быть более эффективным по сравнению с алгоритмом, использующим традиционные гомоморфизмы многоосновных универсальных алгебр. Об этом свидетельствует нижеследующий пример. Для его построения нам потребуются некоторые результаты работы [8].

Пусть $\delta: A_{1} \times A_{2} \times \cdots \times A_{n} \rightarrow A_{0}$ - некоторая операция;

$$
\mathbf{a}=\left(a_{1}, \ldots, a_{n}\right) \in A_{1} \times A_{2} \times \ldots \times A_{n} .
$$

Отображение $L_{\delta, i}(\mathbf{a}): A_{i} \rightarrow A_{0}$ вида

$$
L_{\delta, i}(\mathbf{a})=\delta\left(a_{1}, \ldots, a_{i-1}, x, a_{i+1}, \ldots, a_{n}\right)
$$

называется $i$-й трансляцией операции алгебры $\left(A_{1}, A_{2}, \ldots, A_{n}, A_{0} ; \delta\right)$ относительно а [9].

Говорят [8], что множество отображений $G=\{g \mid g: A \rightarrow B\}$ 2-транзитивно, если для любых пар $\left(a_{1}, a_{2}\right) \in A,\left(b_{1}, b_{2}\right) \in B, a_{1} \neq a_{2}, b_{1} \neq b_{2}$, существует отображение $g \in G$ такое, что $g\left(a_{1}\right)=b_{1}$ и $g\left(a_{2}\right)=b_{2}$.

Предложение 1. ([8]) Пусть $\left(\varepsilon\left(A_{1}\right), \ldots, \varepsilon\left(A_{n}\right), \varepsilon\left(A_{0}\right)\right)$ - конгруэнция на алгебре $\left(A_{1}, \ldots, A_{n}, A_{0} ; \delta\right)$, которой отношение эквивалентности $\varepsilon\left(A_{0}\right)$ разбивает множество $A_{0}$ не менее чем в 2 класса эквивалентности. Тогда, если для некоторого $i \in\{1, \ldots, n\}$ множество $i$-x транслячий 2-транзитивно, то все классъ $[a] \varepsilon\left(A_{i}\right)$ одноточечнь.

Вычислительная сложность алгоритма решения системы уравнений (4) с использованием конгруэнции $\varepsilon=(\varepsilon(A), \varepsilon(B), \varepsilon(C))$ на алгебре $(A, B, C ; F)$ оценивается величиной [4]

$$
\bar{Q}(\varepsilon)=|A / \varepsilon(A)||B / \varepsilon(B)|+\sum\left|\left[a_{s}\right] \varepsilon(A)\right|\left|\left[b_{r}\right] \varepsilon(B)\right|
$$


где суммирование производится по представителям классов $a_{s}, b_{r}$ таким, что

$$
F\left(\left[a_{s}\right] \varepsilon(A),\left[b_{r}\right] \varepsilon(B)\right) \subset[c] \varepsilon(C) .
$$

Таким образом, если отношение эквивалентности $\varepsilon(B)$ тривиально, то вычислительная сложность данного алгоритма решения системы (4) включает перебор всех элементов множества $B$.

Рассмотрим алгебру $\left(S_{B}, B, B ; F\right)$, у которой $S_{B}$ - элементы симметрической группы подстановок множества $B,|B|$ - не простое число, а операция $F$ определяется соотношением

$$
F(g, b)=g(b), \quad \jmath \in S_{B}, \quad b \in B .
$$

Множество 2-х трансляций операции $F$ совпадает с симметрической группой $S_{B}$. Поэтому согласно предложению 1 для любой конгруэнции $\left(\varepsilon_{1}, \varepsilon_{2}, \varepsilon_{3}\right)$ на алгебре $\left(S_{B}, B, B ; F\right)$ такой, что $\varepsilon_{3}$ разбивает множество $B$ не менее чем в 2 класса эквивалентности, отношение эквивалентности $\varepsilon_{2}$ тривиально. В частности, как нетрудно видеть, нетривиальными конгруэнциями на рассматриваемой алгебре будут следующие отношения эквивалентности:

$\varepsilon_{2}=i d-$ отношение равенства;

$\varepsilon_{1}$ - отношение правой смежности группы $S_{B}$ по некоторой интранзитивной подгруппе $G$;

$\varepsilon_{3}$ - отношение принадлежности к орбите (множеству транзитивности) группы $G$.

Вместе с тем на данной алгебре существуют скрещенные конгруэнции, позволяющие построить более эффективный алгоритм решения рассматриваемого уравнения. А именно, пусть $H$ - некоторая импримитивная группа подстановок множества $B$, a $\sigma$ - отношение эквивалентности на $B$, соответствующее разбиению $B$ на блоки импримитивности. Тогда искомая скрещенная конгруэнция имеет вид

$$
\varepsilon=\left(\tau_{H},\left\{\sigma_{[g]}=g^{-1}(\sigma):[g] \in S_{B} / \tau_{H}\right\},\left\{\sigma_{[g]}=\sigma:[g] \in S_{B} / \tau_{H}\right\}\right)
$$

где $\tau_{H}$ - отношение правой смежности в $S_{B}$ по подгруппе $H, g^{-1}(\sigma)$ - отношение эквивалентности на $B$, определяемое следующим образом: $(a, b) \in g^{-1}(\sigma)$ тогда и только тогда, когда $\left(g^{-1}(a), g^{-1}(b)\right) \in \sigma$.

Заметим, что для всех элементов $g \in S_{B}$ из одного смежного класса относительно $\tau_{H}$ отношения эквивалентности $g^{-1}(\sigma)$ совпадают, поэтому скрещенная конгруэнция определена корректно.

Приведенный пример одновременно свидетельствует, что предложение 1 в определенном смысле не распространяется на скрещенные конгруэнции, так как даже максимальное значение транзитивности множества 2-х трансляций не гарантирует отсутствия скрещенных конгруэнций $\left(\varepsilon_{1}, \varepsilon_{2}, \varepsilon_{3}\right)$ с нетривиальными отношениями эквивалентности $\varepsilon_{2}$. Этот пример также показывает, что скрещенные конгруэнции не сводятся к обычным, рассмотренным в работах $[3,4]$.

В [8] было показано, что оптимальное решение уравнений (с точки зрения минимизации вычислений) методом, использующим гомоморфизмы соответствующей многоосновной универсальной алгебры, следует искать среди ее максимальных конгруэнций. Аналогичный результат можно получить и для скрещенных конгруэнций. 
Введем понятие максимальной скрещенной конгруэнции. Заметим, что если на алгебре $(A, B, C ; F)$ существует скрещенная конгруэнция

$$
\varepsilon=\left(\varepsilon_{A},\left\{\varepsilon_{[a]}:[a] \in A / \varepsilon_{A}\right\},\left\{\gamma_{[a]}:[a] \in A / \varepsilon_{A}\right\}\right),
$$

и на основных множествах $A, B$ и $C$ соответственно определена совокупность отношений эквивалентности

$$
\nu=\left(\nu_{A},\left\{\nu_{[a]}:[a] \in A / \nu_{A}\right\},\left\{\mu_{[a]}:[a] \in A / \nu_{A}\right\}\right),
$$

такая, что для любого $a$ выполняются условия

$$
\nu_{A} \subset \varepsilon_{A}, \quad \nu_{[a]} \subset \varepsilon_{[a]}, \quad \gamma_{[a]} \subset \mu_{[a]},
$$

то $\nu$ - также скрещенная конгруэнция. Будем в этом случае говорить, что $\nu$ следствие $\varepsilon$. Отношение следствия задает на множестве всех скрещенных конгруэнций $\operatorname{CrCon}(A, B, C ; F)$ алгебры $(A, B, C ; F)$ отношение частичного порядка.

Определение 5. Максимальные элементы в множестве $\operatorname{Cr} \operatorname{Con}(A, B, C ; F)$, т.е. скрещенные конгруэнции, не являющиеся следствиями, будем называть максимальными скрещенными конгруэнциями.

Предложение 2. Пусть

$$
\varepsilon=\left(\varepsilon_{A},\left\{\varepsilon_{[a]}:[a] \in A / \varepsilon_{A}\right\},\left\{\gamma_{[a]}:[a] \in A / \varepsilon_{A}\right\}\right)
$$

- махсимальная скрещенная конгруэнция на алгебре $(A, B, C ; F)$, a

$$
\nu=\left(\nu_{A},\left\{\nu_{[a]}:[a] \in A / \nu_{A}\right\},\left\{\mu_{[a]}:[a] \in A / \nu_{A}\right\}\right),
$$

- некоторое следствие из $\varepsilon$. Тогда $Q(\varepsilon) \leqslant Q(\nu)$.

Таким образом, оптимальное (с точки зрения минимизации сложности вычислений $(Q(\varepsilon)))$ решение уравнения (4) методом, использующим скрещенные конгруэнции алгебры $(A, B, C ; F)$, следует искать среди ее максимальных конгруэнций.

Доказательство. Так как $\nu_{A} \subset \varepsilon_{A}$ и $\nu_{[a]} \subset \varepsilon_{[a]}$, справедливы неравенства

$$
\left|A / \varepsilon_{A}\right| \leqslant\left|A / \nu_{A}\right|, \quad\left|B / \varepsilon_{[a]}\right| \leqslant\left|B / \nu_{[a]}\right|
$$

и, следовательно,

$$
\sum_{[a] \in A / \varepsilon_{A}}\left|B / \varepsilon_{[a]}\right| \leqslant \sum_{[a] \in A / \nu_{A}}\left|B / \nu_{[a]}\right| .
$$

Пусть для некоторого $(a, b) \in A \times B$

$$
F\left([a] \varepsilon_{A},[b] \varepsilon_{[a]}\right) \subset[c] \gamma_{[a]} .
$$

Тогда, так как $\gamma_{[a]} \subset \mu_{[a]}$, справедливо включение

$$
F\left([a] \varepsilon_{A},[b] \varepsilon_{[a]}\right) \subset[c] \mu_{[a]} .
$$

Следовательно, элементы $a, b$ входят в некоторые классы с представителями соответственно $a_{i}, b_{j}$, для которых $F\left(\left[a_{i}\right] \nu_{A},\left[b_{j}\right] \nu_{[a]}\right) \subset[c] \mu_{[a]}$. Таким образом, и второе слагаемое в выражении (6) для $Q(\varepsilon)$ не больше второго слагаемого в выражении (6) для $Q(\nu)$. Предложение доказано. 
Получающиеся при реализации программных алгоритмов $n$-арные операции, $n>$ 2 , представляют собой, как правило, суперпозицию некоторых бинарных операций. Нетрудно видеть, что алгоритм решения уравнений для таких операций с использованием скрещенных конгруэнций можно построить на основе скрещенных конгруэнций алгебр с бинарными операциями, составляющими указанную суперпозицию. Пусть, например, $n$-арная операция

$$
F: C_{1} \times A_{2} \times \ldots \times A_{n} \rightarrow C_{n}
$$

является суперпозицией $n-1$ бинарных операций

$$
F_{i}: C_{i} \times A_{i+1} \rightarrow C_{i+1}, \quad i=1, \ldots, n-1,
$$

вида

$$
F\left(c_{1}, a_{2}, a_{3}, \ldots, a_{n}\right)=F_{n-1}\left(\ldots F_{2}\left(F_{1}\left(c_{1}, a_{2}\right), a_{3}\right) \ldots, a_{n}\right),
$$

а на алгебрах $\left(C_{i}, A_{i+1}, C_{i+1} ; F_{i}\right)$ существуют скрещенные конгруэнции

$$
E_{i}=\left(\varepsilon_{i}\left(C_{i}\right),\left\{\varepsilon_{i,[c]}:[c] \in C_{i} / \varepsilon_{i}\left(C_{i}\right)\right\},\left\{\gamma_{i,[c]}:[c] \in C_{i} / \varepsilon_{i}\left(C_{i}\right)\right\}\right)
$$

такие, что для любого $i=1, \ldots, n-1$ и любого $[c] \in C_{i} / \varepsilon_{i}\left(C_{i}\right)$ справедливо включение $\gamma_{i,[c]} \subset \varepsilon_{i+1}\left(C_{i+1}\right)$. Тогда использование указанных конгруэнций для решения уравнения

$$
F\left(x_{1}, \ldots, x_{n}\right)=b
$$

сводится к последовательному применению вышеописанного алгоритма решения уравнения (4), то есть сводится к решению уравнений в суперпозициях скрещенных фактор-алгебр вида

$$
F\left(\left[x_{1}\right] \varepsilon_{1}\left(C_{1}\right),\left[x_{2}\right] \varepsilon_{1,\left[x_{1}\right]},\left[x_{3}\right] \varepsilon_{2,\left[y_{1}\right]}, \ldots,\left[x_{n}\right] \varepsilon_{n-1,\left[y_{n-2}\right]}\right) \subset[b] \gamma_{n-1,\left[y_{n-2}\right]},
$$

где $\gamma_{i,[c]} \subset \varepsilon_{i+1}\left(C_{i+1}\right)$ и

$$
\begin{aligned}
F_{1}\left(\left[x_{1}\right] \varepsilon_{1}\left(C_{1}\right),\left[x_{2}\right] \varepsilon_{1,\left[x_{1}\right]}\right) & \subset\left[y_{1}\right] \varepsilon_{2}\left(C_{2}\right), \\
& \cdots \\
F_{i}\left(\left[y_{i-1}\right] \varepsilon_{i}\left(C_{i}\right),\left[x_{i+1}\right] \varepsilon_{i,\left[y_{i-1}\right]}\right) & \subset\left[y_{i}\right] \varepsilon_{i+1}\left(C_{i+1}\right), \\
& \cdots \\
F_{n-1}\left(\left[y_{n-2}\right] \varepsilon_{n-1}\left(C_{n-1}\right),\left[x_{n}\right] \varepsilon_{n-1,\left[y_{n-2}\right]}\right) & \subset[b] \gamma_{n-1,\left[y_{n-2}\right]} .
\end{aligned}
$$

\section{4. Скрещенные конгруэнции некоторых универсальных алгебр}

\section{1. Скрещенные конгруэнции квазигрупп, изотопных группам}

Определение 6. Возьмем некоторое подмножество $X$ в группе $G(\cdot)$. Правой $X$ конгруэнцией на группе $G(\cdot)$ будем называть тройку $\left(X, \varepsilon_{Y}, \varepsilon_{Z}\right)$, где $\varepsilon_{Y}, \varepsilon_{Z}$ - отношения эквивалентности на множестве $G$ такие, что для любого класса эквивалентности $Y_{m}$ относительно $\varepsilon_{Y}$ найдется класс эквивалентности $Z_{k}$ относительно $\varepsilon_{Z}$, для которого будет выполнено соотношение

$$
X \cdot Y_{m} \subset Z_{k}
$$


Аналогично можно определить левые $X$-конгруэнции. Однако в дальнейшем мы будем рассматривать только правые $X$-конгруэнции (без уточнения, что они правые). Полученные результаты естественным образом распространяются на левые $X$-конгруэнции.

Определение 7. Заметим, что если на группе $G(\cdot)$ существует $X$-конгруэнция $\varepsilon=$ $\left(X, \varepsilon_{Y}, \varepsilon_{Z}\right)$ и определены отношения эквивалентности $\varepsilon_{Y}^{\prime}, \varepsilon_{Z}^{\prime}$ такие, что выполняются условия

$$
\varepsilon_{Y}^{\prime} \subset \varepsilon_{Y}, \quad \varepsilon_{Z} \subset \varepsilon_{Z}^{\prime},
$$

то $\varepsilon^{\prime}=\left(X, \varepsilon_{Y}, \varepsilon_{Z}\right)$ - также $X$-конгруэнция на группе $G(\cdot)$. Будем в этом случае говорить, что $\varepsilon^{\prime}-$ следствие $\varepsilon$. $X$-конгруэнцию, не являющуюся следствием, будем называть максимальной $X$-конгруэнцией.

Лемма 2. Если $X$-конгруэнция $\varepsilon=\left(X, \varepsilon_{Y}, \varepsilon_{Z}\right)$ на группе $G(\cdot)$ махсималъна, то вместо включения (9) выполнлетсл равенство

$$
X \cdot Y_{m}=Z_{k} .
$$

Доказательство. В самом деле, если для каких-то классов эквивалентности $Y_{m}$, $Z_{k}$ имеет место строгое включение $X \cdot Y_{m} \subset Z_{k}$, то найдется хотя бы еще один класс $Y_{m^{\prime}}$, для которого также выполнено включение $X_{i} \cdot Y_{m^{\prime}} \subset Z_{k}$. Построим новую конгруэнцию $\widetilde{\varepsilon}$ из $\varepsilon$, объединив в $\varepsilon$ два класса $Y_{m^{\prime}}$ и $Y_{m}$ в один класс $\widetilde{Y}_{m^{\prime \prime}}$. Поскольку конгруэнция $\tilde{\varepsilon}$ строго больше конгруэнции $\varepsilon$, конгруэнция $\varepsilon$ не максимальна, что противоречит условию леммы.

Лемма 3. Если $X$-хонгруэнция $\varepsilon=\left(X, \varepsilon_{Y}, \varepsilon_{Z}\right)$ на группе $G(\cdot)$ максималъна, то умножение на $X$ слева устанавливает взаимно однозначное соответствие между совохупностью классов $Y_{m} u Z_{k}$.

Дожазателъство. Очевидно, что умножение на $X$ - это сюръективное отображение. Покажем, что оно также является инъективным. В самом деле, если бы это было не так, то нашлись бы два класса $Y_{m}$ и $Y_{m^{\prime}}$ такие, что $X \cdot Y_{m}=X \cdot Y_{m^{\prime}}=Z_{k}$, и, следовательно, конгруэнция $\varepsilon$ не была бы максимальной. Таким образом, показана инъективность умножения на $X$ и вместе с тем биективность.

Следствие 1. $X$-хонгруэнция $\varepsilon=\left(X, \varepsilon_{Y}, \varepsilon_{Z}\right)$ на группе $G(\cdot)$ махсималъна тогда и только тогда, когда умножение на $X$ слева устанавливает взаимно однозначное соответствие межсду совокупностью классов $Y_{m} u Z_{k}$.

Лемма 4. При необходимости перенумеровав хлассы $Y_{m}$, можно считать, что для махсимальной $X$-конгруэнции $\left(X, \varepsilon_{Y}, \varepsilon_{Z}\right)$ въполняется равенство

$$
X \cdot Y_{k}=Z_{k}
$$

nрu этом $\left|Y_{k}\right|=\left|Z_{k}\right|$.

Доказательство. Первая часть утверждения леммы следует из леммы 3. Докажем, что $\left|Y_{k}\right|=\left|Z_{k}\right|$. Из равенства $X \cdot Y_{k}=Z_{k}$ следует, что $\left|Y_{k}\right| \leqslant\left|Z_{k}\right|$, но с другой стороны

$$
\sum_{k}\left|Y_{k}\right|=\sum_{k}\left|Z_{k}\right|=|G|
$$

Поэтому для любого $k$ выполняется равенство $\left|Y_{k}\right|=\left|Z_{k}\right|$. 
Предложение 3. Пусть $\left(X, \varepsilon_{Y}, \varepsilon_{Z}\right)-X$-конгруэнция на группе $G(\cdot)$. Тогда для любого элемента $g \in X$ и подгруппы $H=g r\left\langle X \cdot g^{-1}\right\rangle \subset G(\cdot)$ справедливы следующие утверждения:

(1) $X$ включается в некоторый правый смежный класс $H \cdot g$;

(2) любое множество $Z_{k}$ представляется в виде объединения некоторых правых смежных классов группы $G(\cdot)$ по подгруппе $H$.

Дохазательство. Пусть $x_{1} \in X, z_{1} \in Z_{k}$. Выберем класс $Y_{m}$, включающий элемент $x_{1}^{-1} \cdot z_{1}$. Тогда

$$
X \cdot Y_{m} \cap Z_{k} \ni z_{1}
$$

и, согласно определению $X$-конгруэнции, $X \cdot Y_{m} \subset Z_{k}$. Следовательно, для любого $x_{2} \in X$

$$
x_{2} \cdot x_{1}^{-1} \cdot z_{1} \in Z_{k}
$$

и, повторяя выше приведенные рассуждения, получаем, что

$$
x_{3} \cdot x_{1}^{-1} \cdot x_{2} \cdot x_{1}^{-1} \cdot z_{1} \in Z_{k}
$$

для любого $x_{3} \in X$. Аналогично показывается, что

$$
x_{s} \cdot x_{1}^{-1} \cdots x_{2} \cdot x_{1}^{-1} \cdot z_{1} \in Z_{k}
$$

для $x_{1}, x_{2}, \ldots, x_{s} \in X$. Следовательно, $Z_{k}$ включает правый смежный класс группы $G(\cdot)$ по подгруппе $H=g r\left\langle X \cdot x_{1}^{-1}\right\rangle$, т.е. $H \cdot z_{1} \subset Z_{k}$. В силу произвольности $z_{1} \in$ $Z_{k}$ получаем, что класс $Z_{k}$ представляется в виде объединения некоторых правых смежных классов группы $G(\cdot)$ по подгруппе $H$.

Нетрудно заметить также, что $X \subset H \cdot x_{1}$. Положим теперь $g=x_{1}$. Предложение доказано.

Из предложения 3 непосредственно вытекает следующее утверждение.

Предложение 4. Пусть $\left(X_{i}, Y_{m}^{(i)}, Z_{k}^{(i)}\right)$ - схрещеннал конгруэнция на группе $G(\cdot)$. Тогда для любого $i$ существует подгруппа $H_{i} \subset G(\cdot)$ такая, что $X_{i}$ включается $в$ некоторый правый смежсный класс $H_{i} \cdot g$ и любой класс $Z_{k}^{(i)}$ представляетсл в виде объединения правых смежнъх классов по подаруппе $H_{i}$.

Лемма 5. Пусть для трех подмножеств $X, Y, Z$ группы $G(\cdot) \operatorname{maxux,~ито~}|Y|=$ $|Z|$, выполняется равенство $X \cdot Y=Z$. Тогда верно равенство $Y=X^{-1} \cdot Z$, где $X^{-1}=\left\{x^{-1}: x \in X\right\}$.

Дохазательство. Поскольку при любом $x \in X$ выполняются соотношения $x \cdot Y \subset Z$ и $|x \cdot Y|=|Y|=|Z|$, то $x \cdot Y=Z$ и $Y=x^{-1} \cdot Z$. Отсюда следует утверждение леммы.

Следствие 2. Если $\varepsilon=\left(X, Y_{k}, Z_{k}\right)$ - махсимальная $X$-хонгруэнция на группе $G(\cdot)$, то набор множеств $\left(X^{-1}, Z_{k}, Y_{k}\right)$ лөляетсл махсимальной $X^{-1}$-конгруэнцией, которуг далее мъ будем обозначать через $\varepsilon^{-1}$. 
Лемма 6. Пусть $\varepsilon=\left(X, Y_{k}, Z_{k}\right)$ - махсимальная $X$-конгруэнция на әруппе $G(\cdot)$. Tогда существует пара сопряженнъх подгрупn $H \subset G(\cdot) u Q \subset G(\cdot)$, m.e. $g^{-1} \cdot H \cdot g=$ $Q, \operatorname{maxux}$, чmo

(1) $X$ включается в правый смежный класс $H \cdot g$ әруппы $G(\cdot)$ по подәруппе $H$, a $X^{-1}$ включаетсл в правый смежный класс $Q \cdot g^{-1}$ группы $G(\cdot)$ nо подгрyпnе $Q$;

(2) любой класс $Z_{k}^{(i)}$ представляетсл в виде обвединения правых смежных классов группь $G(\cdot)$ по подгруппе $H$, и любой класс $Y_{k}^{(i)}$ представляется в виде объединения правых смежных классов группь $G(\cdot)$ nо подгруппе $Q$.

Доказательство. Поскольку $\left(X, Y_{k}, Z_{k}\right)$ - максимальная $X$-конгруэнция, то, согласно следствию $2,\left(X^{-1}, Z_{k}, Y_{k}\right)$ - тоже максимальная $X$-конгруэнция. Применяя к ним предложение 3 , получаем утверждение леммы.

Пусть $\varepsilon$ - скрещенная конгруэнция на группе $G(\cdot)$. Обозначим через $X_{i}, Y_{m}^{(i)}$, $Z_{k}^{(i)}$ соответствующие классы эквивалентности. Очевидно, что при любом фиксированном $i$ совокупность множеств $\left(X_{i}, Y_{m}^{(i)}, Z_{k}^{(i)}\right)$ образуют $X_{i}$-конгруэнцию на группе $G(\cdot)$, которую мы обозначим через $\varepsilon_{i}$. Заметим, что если скрещенная конгруэнция $\varepsilon$ максимальна, то будут максимальными все $X_{i}$-конгруэнции $\varepsilon_{i}$. Тогда для каждого $i$, согласно следствию 2 , можно построить максимальную $X_{i}^{-1}$-конгруэнцию. Применяя лемму 6 к каждой макисмальной $X_{i}$-конгруэнции $\varepsilon_{i}$, получаем следующую теорему.

Теорема 2. Пусть $\varepsilon=\left(X_{i}, Y_{k}^{(i)}, Z_{k}^{(i)}\right)$ - максималъная скрещенная конгруэнция на әруппе $G(\cdot)$. Тогда для любого $і$ с существует пара сопряженных подгрупп $H_{i} \subset G(\cdot)$ $u Q_{i} \subset G(\cdot)$, m.e. $g_{i}^{-1} \cdot H_{i} \cdot g_{i}=Q_{i}$, maxux, иmo

(1) $X_{i}$ включается в правый смежсный класс $H_{i} \cdot g_{i}$ грyпnы $G(\cdot)$ nо подәруппе $H_{i}$, а $X_{i}^{-1}$ включается в правый смежснй класс $Q_{i} \cdot g_{i}^{-1}$ еруппы $G(\cdot)$ по подгруппе $Q_{i}$

(2) любой класс $Z_{k}^{(i)}$ представляется в виде обвединения правых смежнъх класcoв группы $G(\cdot)$ nо подгруппе $H_{i}$, u любой класс $Y_{k}^{(i)}$ представляется в виде объединения правых смежных классов группы $G(\cdot)$ по подгруппе $Q_{i}$.

Опишем процедуру построения $X$-конгруэнции $\left(X, Y_{k}^{(g)}, Z_{k}^{(g)}\right)$, которую мы будем называть порождающей и обозначать через $\varepsilon^{(g)}$ (смысл названия будет ясен из теоремы 3). Для этого нам понадобится следующее определение.

Определение 8. Пусть $\mathfrak{A}-$ совокупность множеств $A_{s}, \mathfrak{A}=\left\{A_{s}\right\}$. Связной компонентой совокупности $\mathfrak{A}$ будем называть множество $A$, являющееся объединением некоторых множеств $A_{s}$ и удовлетворяющее следующим условиям:

(1) любое множество $A_{s}$ либо целиком лежит в $A$, либо не пересекается с $A$;

(2) для любых двух множеств $A_{s}$ и $A_{s^{\prime}}$, лежащих в $A$, найдется последовательность множеств

$$
A_{s_{1}}=A_{s}, A_{s_{2}}, \ldots, A_{s_{m-1}}, A_{s_{m-1}}=A_{s^{\prime}},
$$

также лежащих в $A$, такая, что $A_{s_{i}} \cap A_{s_{i+1}} \neq \varnothing, i=1, \ldots m-1$. 
Ясно, что любые две связные компоненты совокупности $\mathfrak{A}$ не пересекаются, и объединение всех $A_{s} \in \mathfrak{A}$ разбивается на связные компоненты совокупности $\mathfrak{A}$.

Рассмотрим покрытие группы $G(\cdot)$ совокупностью множеств $X \cdot y$, где $y$ пробегает всю группу $G$. Группа $G$ разбивается на связные компоненты этой совокупности. Теперь в качестве $Z_{k}^{(g)}$ возьмем $k$-ю связную компоненту, а в качестве $Y_{k}^{(g)}$ возьмем множество $Y_{k}^{(g)}=\left\{y: X \cdot y \subset Z_{k}^{(g)}\right\}$. Согласно следствию 1 , порождающая $X$-конгруэнция максимальна.

Предложение 5. Для порождающей $X$-конгруэнщии $\varepsilon^{(g)}$ на әруппе $G(\cdot)$ cуществует nодәрупnа $H \subset G(\cdot)$ maкая, что

(1) $X$ включается в некоторый правый смежный класс $H \cdot y$ группы $G(\cdot)$ по подapynne $H$;

(2) любое множество $Z_{k}^{(g)}$ является правым смежным классом группы $G(\cdot)$ по nodzpynne $H$.

Доказателъство. Согласно предложению 3 существует подгруппа $H \subset G(\cdot)$ такая, что $X$ включается в некоторый правый смежный класс $H \cdot y$. А любое $Z_{k}$ представляется в виде объединения некоторых правых смежных классов группы $G(\cdot)$ по подгруппе $H$, т.е. $\varepsilon(H) \subset \varepsilon_{Z}^{(g)}[X]$, где $\varepsilon(H)$ - отношение эквивалентности на группе $G(\cdot)$, задаваемое разбиением на правые смежные классы по подгруппе $H$, а $\varepsilon_{Z}^{(g)}[X]$ - $Z$-я компонента $X$-конгруэнции $\varepsilon^{(g)}$. Рассмотрим теперь порождающую $(H \cdot y)$ конгруэнцию $\varepsilon^{(g)}[H \cdot y]$. Поскольку $X \subset H \cdot y$,

$$
\varepsilon_{Z}^{(g)}[X] \subset \varepsilon_{Z}^{(g)}[H \cdot y]=\varepsilon(H)
$$

Следовательно, $\varepsilon_{Z}^{(g)}[X]=\varepsilon(H)$.

Пусть множество индексов $J$, которыми заиндексированы классы $Z_{k}^{(g)}$, разбито на множества

$$
J_{1}, \ldots, J_{s}
$$

Образуем новую $X$-конгруэнцию $\left(X, Y_{i}, Z_{i}\right)$ следующим образом:

$$
Z_{i}=\bigsqcup_{k \in J_{i}} Z_{k}^{(g)}, \quad Y_{i}=\bigsqcup_{k \in J_{i}} Y_{k}^{(g)}, \quad i=1, \ldots, s .
$$

Согласно следствию $1, X$-конгруэнция $\left(X, Y_{i}, Z_{i}\right)$ максимальна. Такую процедуру получения из порождающей конгруэнции $\varepsilon^{(g)}$ новых максимальных $X$-конгруэнций назовем объединением классов эквивалентности в $\varepsilon^{(g)}$.

Теорема 3. Любая максимальная $X$-конгруэнция $\varepsilon=\left(X, Y_{i}, Z_{i}\right)$ может быть получена из порождающей $X$-конгруэнции $\varepsilon^{(g)}$ обвединением классов эквивалентнос$m u$.

Доказательство. Покажем, что любой класс $Z_{k}^{(g)}$ целиком лежит в каком-то классе $Z_{i}$. Отсюда будет следовать, что $Y_{k}^{(g)}$ также целиком лежит в соответствующем классе $Y_{i}$. 
Возьмем любые две точки $z^{\prime}, z^{\prime \prime}$ из $Z_{k}^{(g)}$, пусть они принадлежат множествам $X \cdot y^{\prime}, X \cdot y^{\prime \prime} \subset Z_{k}^{(g)}$ соответственно. Если $X \cdot y^{\prime} \cap X \cdot y^{\prime \prime} \neq \varnothing$, то оба множества $X \cdot y^{\prime}$, $X \cdot y^{\prime \prime}$ обязаны целиком лежать в каком-то классе $Z_{i}$. Следовательно, $z^{\prime}, z^{\prime \prime} \in Z_{i}$. Если же $X \cdot y^{\prime} \cap X \cdot y^{\prime \prime}=\varnothing$, то, согласно процедуре построения множеств $Z_{k}^{(g)}$, найдется последовательность множеств

$$
X \cdot y_{1}=X \cdot y^{\prime}, X \cdot y_{2}, \ldots, X \cdot y_{s-1}, X \cdot y_{s}=X \cdot y^{\prime \prime}
$$

также лежащих в $Z_{k}^{(g)}$, такая, что

$$
X \cdot y_{i} \cap X \cdot y_{i+1} \neq \varnothing, \quad i=1, \ldots s-1
$$

Каждое следующее множество в этой последовательности целиком лежит в том же классе $Z_{i}$, что и предыдущее, поэтому $X \cdot y^{\prime}, X \cdot y^{\prime \prime} \subset Z_{i}$. Следовательно, в этом случае также $z^{\prime}, z^{\prime \prime} \in Z_{i}$.

Итак, любой класс $Z_{i}$ является объединением каких-то классов $Z_{k}^{(g)}$, в свою очередь соответствующий класс $Y_{i}$ является объединением соответствующих классов $Y_{k}^{(g)}$.

Определение 9. Напомним [10], что квазигруппа $G(*)$ называется изотопной группе $G(\cdot)$, если существуют такие подстановки $\alpha, \beta, \gamma$ на множестве $G$, что для всех $x, y \in G$

$$
x * y=\gamma^{-1}(\alpha x \cdot \beta y)
$$

Легко видеть, что имеет место следующая лемма.

Лемма 7. Пусть квазигруппа $G(*)$ изотопна группе $G(\cdot)$ при изотопии $(\alpha, \beta, \gamma) u$ $\varepsilon=\left(X_{i}, Y_{k}^{(i)}, Z_{k}^{(i)}\right)$ - махсимальная скрещенная конгруэнция на квазигруппе $G(*)$. Тогда $\widetilde{\varepsilon}=\left(\alpha\left(X_{i}\right), \beta\left(Y_{k}^{(i)}\right), \gamma\left(Z_{k}^{(i)}\right)\right.$ - махсимальная скрещенная конгруэнция на групne $G(\cdot)$.

Из теоремы 2 и леммы 7 вытекает следующая теорема.

Теорема 4. Пусть $\varepsilon=\left(X_{i}, Y_{k}^{(i)}, Z_{k}^{(i)}\right)$ - максимальная скрещеннал конгруэнция на квазигруппе $G(*)$, изотопной группе $G(\cdot)$ при изотопии $(\alpha, \beta, \gamma)$. Тогда для любого $i$ существует пара сопряженных подерупп $H_{i} \subset G(\cdot)$ u $Q_{i} \subset G(\cdot)$, m.e. $g_{i}^{-1} \cdot H_{i} \cdot g_{i}=$ $Q_{i}, \operatorname{maxux}$, чmo

(1) $\alpha\left(X_{i}\right)$ включается в правый смежсный класс $H_{i} \cdot g_{i}$ группь $G(\cdot)$ по подгруппе $H_{i}$, a $\alpha\left(X_{i}\right)^{-1}$ включается в правъй смежный класс $Q_{i} \cdot g_{i}^{-1}$ әруппъ $G(\cdot)$ по nodzpynne $Q_{i}$;

(2) любое множество $\gamma\left(Z_{k}^{(i)}\right)$ представляется в виде обтединения правъх смежных классов группы $G(\cdot)$ по подгруппе $H_{i}$, а любое множество $\beta\left(Y_{k}^{(i)}\right)$ представляется в виде объединения правых смежных классов группы $G(\cdot)$ по подapynne $Q_{i}$. 


\section{2. Конгруэнции и скрещенные конгруэнции квазигрупп, скрещенно изотопных группам}

Теорема 5. Пусть квазигруппа $G(*)$ схрещенно изотопна группе $G(\cdot)$ при скрещенной изотопии $(e, B, e)$, где $е$ - тождественная подстановка на множестве G. Рассмотрим скрещенную хонгруэнцию $\varepsilon=\left(X_{i}, Y_{m}^{(i)}, Z_{k}^{(i)}\right)$ на квазигруппе $G(*)$, согласованную со схрещенной изотопией $(e, B, e)$, это означает, что для любой пары классов $X_{i}, Y_{m}^{(i)}$ найдется класс $Z_{k}^{(i)}$, удовлетворяющий соотношению $X_{i} \cdot B\left(X_{i}, Y_{m}^{(i)}\right) \subset Z_{k}^{(i)}$. Тогда для каждого $i$ cyществует подгруппа $H_{i} \subset G(\cdot)$ maкая, чmo

(1) $X_{i}$ включается в некоторый правъй смежнъй класс $H_{i} \cdot g$ әруппь $G(\cdot)$ по nodzpynne $H_{i}$;

(2) любое множество $Z_{k}^{(i)}$ представляется в виде обвединения правых смежсных классов группь $G(\cdot)$ nо подгрупnе $H_{i}$.

При этом, если для фихсированного $і$ разбиение множества $G$ на классы $Z_{k}^{(i)}$ состоит не менее чем из 2 элементов, то подгруппа $H_{i}$ - собственная.

Доказателъство. Рассмотрим совокупность множеств $B_{m}^{(i)}=B\left(X_{i}, Y_{m}^{(i)}\right)$. Поскольку $B$ является обратимой справа операцией, при любом фиксированном $i$ множества $B_{m}^{(i)}$ образуют покрытие множества $G$, т.е.

$$
\bigcup_{m} B_{m}^{(i)}=G
$$

Обозначим через $\bar{B}_{s}^{(i)}$ связные компоненты этого покрытия. Тогда совокупность множеств $\left(X_{i}, \bar{B}_{s}^{(i)}, Z_{k}^{(i)}\right)$ образует скрещенную конгруэнцию на группе $G(\cdot)$. Применяя предложение 3 ,получаем утверждение теоремы.

Эта теорема легко может быть переформулирована и для скрещенных изотопий общего вида. В общем случае строение классов $Y_{m}^{(i)}$ скрещенных конгруэнций квазигрупп, скрещенно изотопных группам, зависит от вида операции $B$. Об этом свидетельствует следующий пример.

Рассмотрим квазигруппу $G(\Phi)$, скрещенно $R$-изотопную абелевой группе $G(\cdot)$ вида

$$
\Phi(x, y)=x \cdot B(x, y),
$$

такую, что операция $B$ на множестве $G$ есть квазигруппа, $L$-изотопная некоторой группе $G(*)$, т.е.

$$
\Phi(x, y)=x \cdot \gamma^{-1}(A(x, y) * \beta(y))
$$

где $S=(A, \beta, \gamma)-L$-изотопия.

Такие квазигруппы существуют. Например, если в преобразовании $F$, определенном во введении формулой (1), функция $f$ является операцией квазигруппы $V_{n}(f)$, изотопной группе $V_{n}(\circ)$, то нетрудно показать, что операция $F$ имеет вид $(10)$

$$
\begin{aligned}
F\left(\left(L_{i-1}, R_{i-1}\right),\left(K_{i}, K_{i+1}\right)\right) & \\
& =\left(L_{i-1}, R_{i-1}\right) \oplus \bar{\gamma}^{-1}\left(A\left(\left(L_{i-1}, R_{i-1}\right),\left(K_{i}, K_{i+1}\right)\right) \overline{\bar{\alpha}} \bar{\beta}\left(K_{i}, K_{i+1}\right)\right),
\end{aligned}
$$


где

$$
\begin{aligned}
A\left(\left(L_{i-1}, R_{i-1}\right),\left(K_{i}, K_{i+1}\right)\right) & =\bar{\alpha}\left(R_{i-1}, L_{i-1} \oplus f\left(R_{i-1}, K_{i}\right)\right), \\
f(a, b) & =\gamma^{-1}(\alpha(a) \circ \beta(b)), \\
\bar{\gamma}^{-1}(a, b) & =\left(\gamma^{-1}(a), \gamma^{-1}(b)\right), \\
\bar{\beta}(a, b) & =(\beta(a), \beta(b)), \\
\bar{\alpha}(a, b) & =(\alpha(a), \alpha(b)), \\
(a, b) \overline{\mathrm{o}}(c, d) & =(a \circ c, b \circ d), \quad a, b, c, d \in V_{n} .
\end{aligned}
$$

Пусть $\left(X_{i}, Y_{j}, Z_{k}\right)$ - конгруэнция на квазигруппе $G(\Phi)$, согласованная с двумя скрещенными изотопиями $(e, B, e)$ и $(A, \beta, \gamma)$. Это означает, что для любых классов $X_{i}, Y_{j}$ существует класс $Z_{k}$, удовлетворяющий соотношению

$$
X_{i} \cdot \gamma^{-1}\left(A\left(X_{i}, Y_{j}\right) * \beta\left(Y_{j}\right)\right) \subset Z_{k}
$$

Положим $B_{j}^{i}=\gamma^{-1}\left(A\left(X_{i}, Y_{j}\right) * \beta\left(Y_{j}\right)\right)$.

Лемма 8. При любом фихсированном $i$ имеет место равенство

$$
\bigcup_{j} B_{j}^{i}=G
$$

При любом фиксированном $j$ имеет место равенство

$$
\bigcup_{i} B_{j}^{i}=G
$$

Доказательство. При любом фиксированном $x$ из того, что $B-$ квазигруппа, вытекает равенство

$$
\bigcup_{y \in G} B(x, y)=G .
$$

Из этого равенства следует первое утверждение леммы.

Докажем теперь второе утверждение. Заметим, что равенство $\bigcup_{i} B_{j}^{i}=G$ эквивалентно равенству $\bigcup_{i} \gamma\left(B_{j}^{i}\right)=G$. Возьмем любой элемент $g \in G$ и любой элемент $y \in Y_{j}$. Рассмотрим уравнение $A(x, y)=[\beta(y)]^{-1} * g$ относительно $x$. Поскольку $A$ обратима слева, существует $x \in X_{i}$, удовлетворяющий этому уравнению. Таким образом, для любого $g \in G$ найдется некоторое множество $\gamma\left(B_{j}^{i}\right)$ такое, что $g \in \gamma\left(B_{j}^{i}\right)$.

Лемма 9. Пусть данъ

$$
\bigsqcup_{i \in I} X_{i}, \quad \bigsqcup_{k \in K} Z_{k}
$$

два разбиения произволъной конечной группљ $G(\cdot)$ на классы эквивалентности, совокупность множеств $B_{j}^{i}$, образующих при фиксированном $i$ покрытие группы $G$, m.e.

$$
G=\bigcup_{j} B_{j}^{i},
$$

и для любой пары $X_{i}, B_{j}^{i}$ найдется класс $Z_{k}$ такой, что $X_{i} \cdot B_{j}^{i} \subset Z_{k}$. 
Тогда классы эквивалентности $X_{i}$ являются подмножествами в правых смежных классах группы $G(\cdot)$ по некоторой подгруппе $H$, а классы эквивалентности $Z_{k}$ представляют собой обвединение некоторых правых смежных классов группы $G(\cdot)$ no nodzpynne $H$.

Доказательство. Пусть $x_{1} \in X_{i_{1}}, z_{1} \in Z_{k}$. Выберем подмножество $B_{j}^{i_{1}}$, включающее элемент $x_{1}^{-1} \cdot z_{1}$. Тогда

$$
X_{i_{1}} \cdot B_{j}^{i_{1}} \cap Z_{k} \ni z_{1},
$$

т.е. $X_{i_{1}} \cdot B_{j}^{i_{1}} \subset Z_{k}$. Следовательно, для любого $x_{2} \in X_{i_{1}}$

$$
x_{2} \cdot x_{1}^{-1} \cdot z_{1} \in Z_{k}
$$

Последовательно повторяя вышеприведенные рассуждения, получаем, что для любой последовательности пар $x_{1}, x_{2} \in X_{i_{1}}, x_{3}, x_{4} \in X_{i_{2}}, \ldots, x_{2 s-1}, x_{2 s} \in X_{i_{s}}$ имеет место соотношение $x_{2 s} \cdot x_{2 s-1}^{-1} \cdot \ldots \cdot x_{4} \cdot x_{3}^{-1} \cdot x_{2} \cdot x_{1}^{-1} \in Z_{k}$. Следовательно, класс $Z_{k}$ содержит правый смежный класс $H \cdot z_{1}$ группы $G(\cdot)$ по подгруппе

$$
H=g r\left\langle X_{i} \cdot x_{t}^{-1} ; x_{t} \in X_{i}, i \in I\right\rangle \text {. }
$$

Таким образом, любой класс $Z_{k}$ в силу произвольности элемента $z_{1} \in Z_{k}$ является объединением некоторых правых смежных классов группы $G(\cdot)$ по подгруппе $H$.

Легко также видеть, что любой класс $X_{i}$ является подмножеством смежного класса $H \cdot x, x \in X_{i}$.

Лемма 10. Пусть в условиях предыдущей леммы 9 группа $G(\cdot)$ абелева, и классы $Z_{k}$ являются подмножествами в нехоторых смежных классах группы $G(\cdot)$ по некоторой подгруппе $H_{0}$. Тогда $H \subset H_{0}$ и множества $B_{j}^{i}$ являются подмножествами в смежных классах группы $G(\cdot)$ по подгруппе $H_{0}$.

Доказательство. Из соотношения $X_{i} \cdot B_{j}^{i} \subset Z_{k}$ вытекает, что $B_{j}^{i} \subset X_{i}^{-1} \cdot Z_{k}$, но

$$
X_{i}^{-1} \subset H \cdot x^{-1} \subset H_{0} \cdot x^{-1}, \quad x \in X_{i}, \quad Z_{k} \subset H_{0} \cdot z, \quad z \in Z_{k},
$$

поэтому $B_{j}^{i} \subset H_{0} \cdot x^{-1} \cdot z$.

Заметим, что указанные в лемме 10 скрещенные конгруэнции представляют интерес с точки зрения построения алгоритмов решения уравнений вида (8) над алгебрами, $n$-арные операции которых представляют собой суперпозиции рассматриваемых квазигрупп.

Следствие 3. Если в условиях леммы 10 подгруппа $H_{0}$ собственная, то при любом $j$ разбиение похрытия

$$
G=\bigcup_{i} B_{j}^{i}
$$

на связные компоненты не тривиалъно.

Рассмотрим совокупность множеств $\left(A_{j}^{i}, \beta\left(Y_{j}\right), B_{j}^{i}\right)$, где $A_{j}^{i}=A\left(X_{i}, Y_{j}\right)$. Эти множества связаны соотношением

$$
A_{j}^{i} * \beta\left(Y_{j}\right)=\gamma\left(B_{j}^{i}\right)
$$


Заметим, что поскольку $A$ обратима слева, при любом фиксированном $j$ объединение $\bigcup_{i} A_{j}^{i}$ является покрытием группы $G(*)$. Обозначим через $\bar{B}_{j}^{s}$ связные компоненты покрытия $G=\bigcup_{i} B_{j}^{i}$, а через $\bar{A}_{j}^{s}$ соответствующие связные компоненты покрытия $G=\bigcup_{i} A_{j}^{i}$. Пусть, как и в условиях леммы 10 ,существует подгруппа $H_{0} \subset G(\cdot)$ такая, что классы $Z_{k}$ являются подмножествами в некоторых смежных классах группы $G(\cdot)$ по подгруппе $H_{0}$. Тогда любое множество $B_{j}^{i}$, а значит, и любая связная компонента $\bar{B}_{j}^{s}$, содержится в некотором смежном классе $H_{0} \cdot b_{k}$. Следовательно, учитывая равенство (11), можно утверждать, что совокупность множеств $\left(\bar{A}_{j}^{s}, \beta\left(Y_{j}\right), \gamma\left(H_{0} \cdot b_{k}\right)\right)$ удовлетворяет условиям леммы 9 . Теперь применяя эту лемму, получаем следующее утверждение.

Предложение 6. В условиях леммъ 10 существует подгруппа $Q \subset G(*)$ такая, что множество $\beta\left(Y_{j}\right)$ является подмножеством в нехотором левом смежном классе $y_{j} * Q$, т.е. $\beta\left(Y_{j}\right) \subset y_{j} * Q$, и множество $\gamma\left(\bar{B}_{j}^{i}\right)$ является объединением некоторых левых смежных классов группы $G(*)$ по подгруппе $Q$.

Предложение 7. Если хонгруэнция $\left(X_{i}, Y_{j}, Z_{k}\right)$ такова, ито $H_{0}$ из леммъ 10 собственная подеруппа в $G(\cdot)$, то сопутствующие подгруппь $H(\cdot)$ u $Q(*)$ также собственные соответственно в $G(\cdot)$ u $G(*)$.

Доказательство. Поскольку $H \subset H_{0}$, то $H(\cdot)$ - также собственная подгруппа в $G(\cdot)$.

Согласно следствию 3 , если $H_{0}$ собственная подгруппа, то при любом $j$ в покрытии $\bigcup_{i} \gamma\left(B_{j}^{i}\right)$ по крайней мере две связные компоненты $\gamma\left(\bar{B}_{j}^{s}\right)$. Тогда из второго пункта предложения 6 следует, что $Q(*)$ не может совпадать со всей группой $G(*)$.

\section{Список литературы}

1. Белоусов В. Д., Скрещенные изотопии квазигрупп. Квазигруппы и их системъ, Математические исследования. (1990) 113, 14-20.

2. Schneier B., Applied Cryptography. Protocols, Algorithms and Source Code in C. Wiley, New York, 1996.

3. Higgins Ph. J., Algebras with a scheme of operators. Math. Nachr. (1963) 27, 115-132.

4. Горчинский Ю. Н., О гомоморфизмах многоосновных универсальных алгебр в связи с криптографическими применениями. Труды по дискретной математике (1997) 1, 67-84.

5. Hellman M., Merkle R., Schroeppel R., Waschington L., Diffie W. Results of an initial attemp to cryptanalyze the NBS Data Encryption Standard. Inform. Syst. Lab. Rep. SEL 76042, Stanford Univ., 1976.

6. Meyer C. H., Ciphertext/plaintext and ciphertext/key dependencies vs. number of rounds for the data encryption standard. In: AFJPS Conf. Proc. 1978, p. 47.

7. Chaurn D., Evertse J. H., Cryptanalysis of DES with reduced number of rounds. Sequences of linear factors in block ciphers Lect. Notes in Comput. Sci. (1986) 218, 192-211.

8. Шапошников И. Г., О конгруэнциях конечных многоосновных универсальных алгебр Дискретная математика (1999) 11, N3, 48-62.

9. Кон П., Универсальная алгебра. Мир, Москва, 1968.

10. Белоусов В. Д., Основљ теории квазигрупп и луп. Наука, Москва, 1967.

Статья поступила 14.04.1999. 